\title{
Simulation of an Asymmetric Transvers Electric TE
}

\section{Metamaterial Absorber}

\author{
H. M. Mousa ${ }^{1}$, M. M. Shabat ${ }^{2}$ and D. M. Schaadt ${ }^{3}$ \\ 1. Physics Department, Al Azhar University, Gaza, Gaza Strip, Palestinian Authority \\ 2. Physics Department, Islamic University, Gaza, P.O. Box 108, Gaza Strip, Palestinian Authority \\ 3. Institute of Energy Research and Physical Technologies, Clausthal University of Technology, Leibnizstr. 4, 38678 Clausthal \\ -Zellerfeld, Germany
}

\begin{abstract}
This paper introduces the principle method and simulation of an asymmetric TE (transverse electric) mode absorption in a lossy artificial metamaterial (LHM (left-handed material)). LHM is sandwiched between a lossy substrate and covered by a lossless dielectric cladding. The asymmetry solutions of the eigenvalue equation describe lossy-guided modes with complex-valued propagation constants. The dispersion relations, normalized field and the longitudinal attenuation were numerically solved for a given set of parameters: frequency range; film's thicknesses; and TE mode order. We found that high order modes, which are guided in thinner films, generally have more loss of power than low-order modes since the mode attenuation along z-axis $\alpha_{z}$ increases to negative values as the mode's number increases, and the film thickness decreases. Moreover, for LHM, at incident wavelength $=1.9$ $\mu m$, refractive index $=-3.74+\mathrm{i} 2$ and at thickness $=0.3 \mu \mathrm{m}$, the modes of order $(4,5,6)$ attain high positive attenuation which means these modes have larger absorption lengths and they are better absorber than the others. This LHM is appropriate for solar cell applications. For arbitrary LHM, at frequency band of wavelengt (600, 700 to $900 \mathrm{~nm})$, the best absorption is attained at longer wavelengths and for lower order modes at wider films. The obtained results could be useful for the design of future light absorbers.
\end{abstract}

Key words: Attenuation, Eigen-value equation, left handed material, solar cells.

\section{Introduction}

Recently, there has been an increasing growth and a subject of intense interest of new artificial materials called Metamaterials or LHM (left-handed materials) [1]. The increasing interest comes from the unusual properties of these materials as the term LHM stems from the left-handed orientation of the triad directions of the electric field, the magnetic field, and the wave vector. In LHM, the wave vector is in the opposite direction of the Poynting vector. On the other hand, in a normal RHM (right-handed material), the wave vector and the Poynting vector are in the same direction [2]. LHMs are composite materials, structured at sub-wavelength scales, which give rise to electromagnetic resonances. Due to the resonant

Corresponding author: M. M. Shabat, Ph.D., Professor Dr., research field: optoelectronics. behavior of LHMs, LHMs can exhibit extreme values of the effective medium parameters such as large and negative dielectric permittivity and permeability [3] which usually renders them dissipative and can be scaled from microwave [4] and terahertz [5, 6] through the infrared $[7,8]$ almost into optical frequencies [10]. Optimized metamaterials with high absorption have been proposed for applications such as thermal spatial light modulators [11], plasmonic sensors [12, 13], thermal bolometers [14], anti-reflection coatings [15] and solar thermo-photovoltaic [16]. The goal of photovoltaic structures is to absorb as much light as possible within specific layers. The metamaterial-based electromagnetic absorbers are promising candidates for solar energy harvesting with improved efficiency, wider solar spectrum, and lower material consumption, 
and are attracting widespread interest. The first perfect metamaterial absorber, having the measured absorptivity of about $88 \%$, composed of a metallic split ring and a cut wire separated by a dielectric layer was demonstrated by Ref. [19]. Several efforts have been made on terahertz metamaterial absorbers $[8,9$, 14]. As photovoltaic device thicknesses continue to shrink and nanostructured designs emerge where their feature sizes reach the order of $1.0 \mu \mathrm{m}$ or less, geometric optics no longer provides an accurate description of field propagation at optical wavelengths. It is therefore an important goal to better understand the problem of waveguide in lossy dielectric films [20, 21]. They explored lossy mode propagation in the context of photovoltaic by modeling a thin film solar cell made of an amorphous silicon, (RHM) with a symmetric and an asymmetric three layer slab. These modes have tremendous longitudinal attenuation, making them especially interesting for the study of thin film photovoltaic. The solutions are applicable for the generalized three-layer dielectric waveguide under any arbitrary combination of gain or lossy materials. This paper examines the problem of lossy waveguide absorption when a LHM is implemented as lossy thin film in an asymmetric waveguide, as depicted in Fig. 1. It consists of LHM slab with thickness $2 h$ covered by a lossless dielectric cladding of real refractive index $n_{c}$. The substrate is lossy with complex index of $\widetilde{n}_{s}=n_{s}+i k_{s}$. LHM film is also described by a complex index of refraction $\tilde{n}_{h}=-\sqrt{\mu_{h} \varepsilon_{h}}+i k_{h}$. The simulations are performed for a metamaterial scaled over near-infrared wavelength range [22]. It consists of a pair of gold films separated by a dielectric layer with a two dimensional square periodic array of circular holes performing the entire multilayer structure. The negative refractive index was obtained at a wavelength around $2000 \mathrm{~nm}$, the real part is -2 . Furthermore, the proposed structure has a minimum feature size of $\sim 100 \mathrm{~nm}$. The simulations are also performed for another arbitrary LHM which has negative index in the visible region of frequency band at wavelength of value $(600,700$ to $900 \mathrm{~nm})$.

\section{The Mathematical Formulation and Structure Model}

To better understand the unusual nature of the Metamaterials or LHM in the tri-layered waveguide structure, the propagation of TE (transverse electric) waves through a thin lossy film of LHM with thickness $2 h$ covered by a lossless cladding is considered. LHM film occupies the region $-h \prec x \prec h$. The cladding occupies the region $x \succ h$, and the substrate in the region $x \prec-h$.

We present the eigenvalue equation for TE waves propagating in the $\mathrm{z}$ direction with a propagation wave constant expressed in the phasor form of $\exp \left[i\left(k_{z} z-2 \pi f t\right)\right], f$ is the operating frequency. The electric and magnetic field vectors for TE waves propagating along $\mathrm{z}$-axis with angular frequency $\omega$ and wavenumber $k_{z}$ are defined as in Refs. [23, 24]:

$$
\begin{aligned}
& E=\left[0, E_{y}(\omega, z), 0\right] \exp i\left(k_{z} z-\omega t\right) \\
& H=\left[H_{x}(\omega, z), 0, H_{z}(\omega, z)\right] \exp i\left(k_{z} z-\omega t\right)
\end{aligned}
$$

In lossy LHM film, $-h \prec x \prec h$

The wave equation can be found easily from the Maxwell's equations as:

$$
\frac{\partial^{2} E_{y}}{\partial x^{2}}-\left(k_{z}^{2}+k_{h}^{2}\right) E_{y}=0
$$

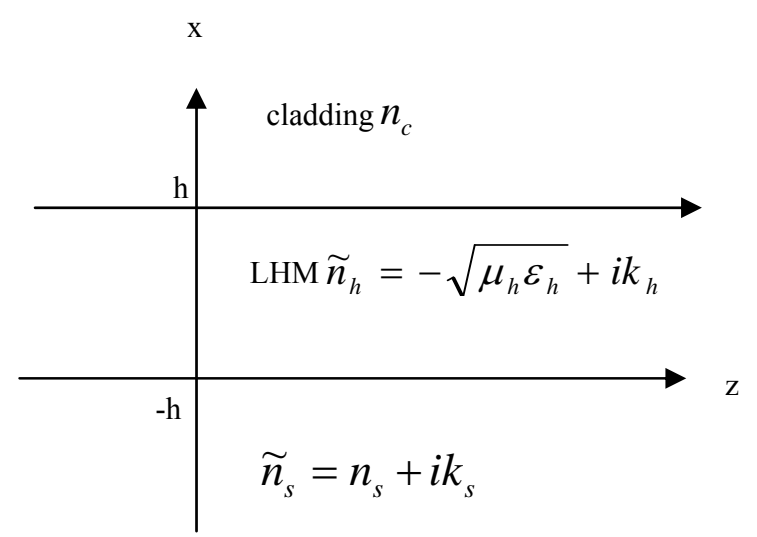

Fig. 1 Waveguide configuration for a lossy asymmetry LHM waveguide. 
where $k_{h}=k_{0} n_{h} \quad$ and, $\quad \tilde{n}_{h}=-\sqrt{\mu_{h} \varepsilon_{h}}+i k \quad$ is refractive index of LHM film. $\varepsilon_{h}, \mu_{h}$ is the electric permittivity and magnetic permeability of LHM, respectively. $k_{z}=\beta_{z}+i \alpha_{z}$, where $\beta_{z}$ is the longitudinal phase constant, $\alpha_{z}$ is the mode attenuation coefficient along z-axis, $k_{0}=2 \pi / \lambda$ is the wave propagation length in free space and $\lambda$ is the free-space wavelength for the model.

The asymmetric solution of Eq. (2) has the form [20]:

$$
\begin{gathered}
E_{y}=E_{0} e^{i k_{z} z}\left\lfloor e^{i k_{x} x}+B e^{-i k_{x} x}\right\rfloor, \\
H_{z}=\left(1 / \omega \mu_{0} \mu_{h}\right) E_{0} k_{x} e^{i k_{z} z}\left\lfloor e^{i k_{x} x}-B e^{-i k_{x} x}\right\rfloor
\end{gathered}
$$

If this solution is substituted into Eq. (2), the resulted relation is

$$
k_{h}^{2}=k_{x}^{2}+k_{z}^{2}
$$

In lossless cladding, $x \succ h$

The wave equation is:

$$
\frac{\partial^{2} E_{y}}{\partial x^{2}}+\left(k_{c}^{2}-k_{z}^{2}\right) E_{y}=0
$$

where, $k_{c}=k_{0} n_{c}$ is the wave number of the cladding region.

The asymmetric solution for Eq. (6) is given by Ref. [20]

$$
\begin{gathered}
E_{y}=A E_{0} e^{i k_{z} z} e^{i \gamma_{c}(x-h)} \\
H_{z}=\left(\gamma_{c} / \omega \mu_{0}\right) E_{0} A e^{i k_{z} z} e^{i \gamma_{c}(x-h)}
\end{gathered}
$$

$A$ is a constant determined by boundary conditions and $\gamma_{c}$ is the complex propagation constant, the real component of $\gamma$ causes the phase oscillation with respect to $\mathrm{x}$-axis. It satisfies the relation

$$
k_{c}^{2}=k_{z}^{2}+\gamma_{c}^{2}
$$

In lossy substrate, $x \prec-h$

The wave equation is:

$$
\frac{\partial^{2} E_{y}}{\partial x^{2}}+\left(k_{s}^{2}-k_{z}^{2}\right) E_{y}=0
$$

where, $k_{s}=k_{0} n_{s}$ is the wave number of the substrate region.

The asymmetric solution for Eq. (10) is given by

$$
\begin{gathered}
E_{y}=C E_{0} e^{i k_{z} z} e^{-i \gamma_{s}(x+h)} \\
H_{z}=-\left(\gamma_{s} / \omega \mu_{0}\right) E_{0} C e^{i k_{z} z} e^{-i \gamma_{s}(x+h)}
\end{gathered}
$$

$\gamma_{s}$ is the complex propagation constant. It satisfies the following relation

$$
k_{s}^{2}=k_{z}^{2}+\gamma_{s}^{2}
$$

The continuity of $E_{y}$ and $H_{Z}$ at the boundary $x=-h$ leads to the following equations

$$
\begin{gathered}
e^{-i k_{x} h}+B e^{i k_{x} h}=C \\
e^{-i k_{x} h}-B e^{i k_{x} h}=-\gamma_{s} C \mu_{h} / k_{x}
\end{gathered}
$$

The continuity of $E_{y}$ and $H_{Z}$ at the boundary $x=h$ leads to the following equations

$$
\begin{gathered}
e^{i k_{x} h}+B e^{-i k_{x} h}=A \\
e^{i k_{x} h}-B e^{-i k_{x} h}=\gamma_{c} A / k_{x}
\end{gathered}
$$

Using a little manipulation and substitution, the TE asymmetric eigenvalue equation is obtained as:

$$
e^{i 4 k_{x} h}=\frac{\left(k_{x}+\gamma_{s} \mu_{h}\right)\left(k_{x}+\gamma_{c}\right)}{\left(k_{x}^{2}-k_{x} \gamma_{c}-\mu_{h} \gamma_{s} k_{x}+\mu_{h} \gamma_{s} \gamma_{c}\right)}
$$

And

$$
B=\frac{\left(k_{x}-\gamma_{c}\right) e^{2 i k_{x} h}}{\left(k_{x}+\gamma_{c}\right)}
$$

By Eqs. (5), (9) and (13),

$$
\begin{aligned}
& \gamma_{c}=\sqrt{k_{c}^{2}-k_{h}^{2}+k_{x}^{2}} \\
& \gamma_{s}=\sqrt{k_{s}^{2}-k_{h}^{2}+k_{x}^{2}}
\end{aligned}
$$

Eq. (18) determines the allowed values for the TE complex wave number of asymmetric modes. Since the eigenvalue equation is transcendental, it can only be solved through iterative methods. We used steepest descent method with linear line search [18, 19]. 
Values of $k_{x}$ which satisfy the eigenvalue Eq. (18) can then back-substituted to solve for all other propagation constants and generate the total TE field solutions over all space.

\section{Numerical Results and Discussion}

In our computation and simulation, the following data has been used in near infrared frequencies, such as $160 \mathrm{THz} \quad(\lambda=1.9 \mu \mathrm{m})$. LHM has $\varepsilon_{h}=-14, \mu_{h}=-1 \quad[22]$ and refractive index $\widetilde{n}_{h}=-\sqrt{\varepsilon_{h} \mu_{h}}+i 2=-3.74+i 2$, the cladding refractive index $n_{c}=1$ and substrate refractive index $\widetilde{n}_{s}=1.26+i 7.2$. The dispersion Eq. (18) has been solved to compute the complex wave numbers of the modes at the film thickness $h=0.5 \mu \mathrm{m}$, Fig. 2 displays the corresponding electric field profile (normalized to unit amplitude $E_{0}$ ) for the modes of lossy LHM waveguide (i.e. $M=0, M=1$ and $M=4$ ). The amplitude $E_{0}$ is to be supposed $1 \mathrm{~V} / \mathrm{m}$. The resultant propagation constants are summarized in Table 1. We notice that through the LHM film $(-0.5 \prec x \prec 0.5)$ the normalized electric field is trapped and decreases from 1.8 to 0.7 by the mode's order increase from 0 to 1 , respectively. This is because

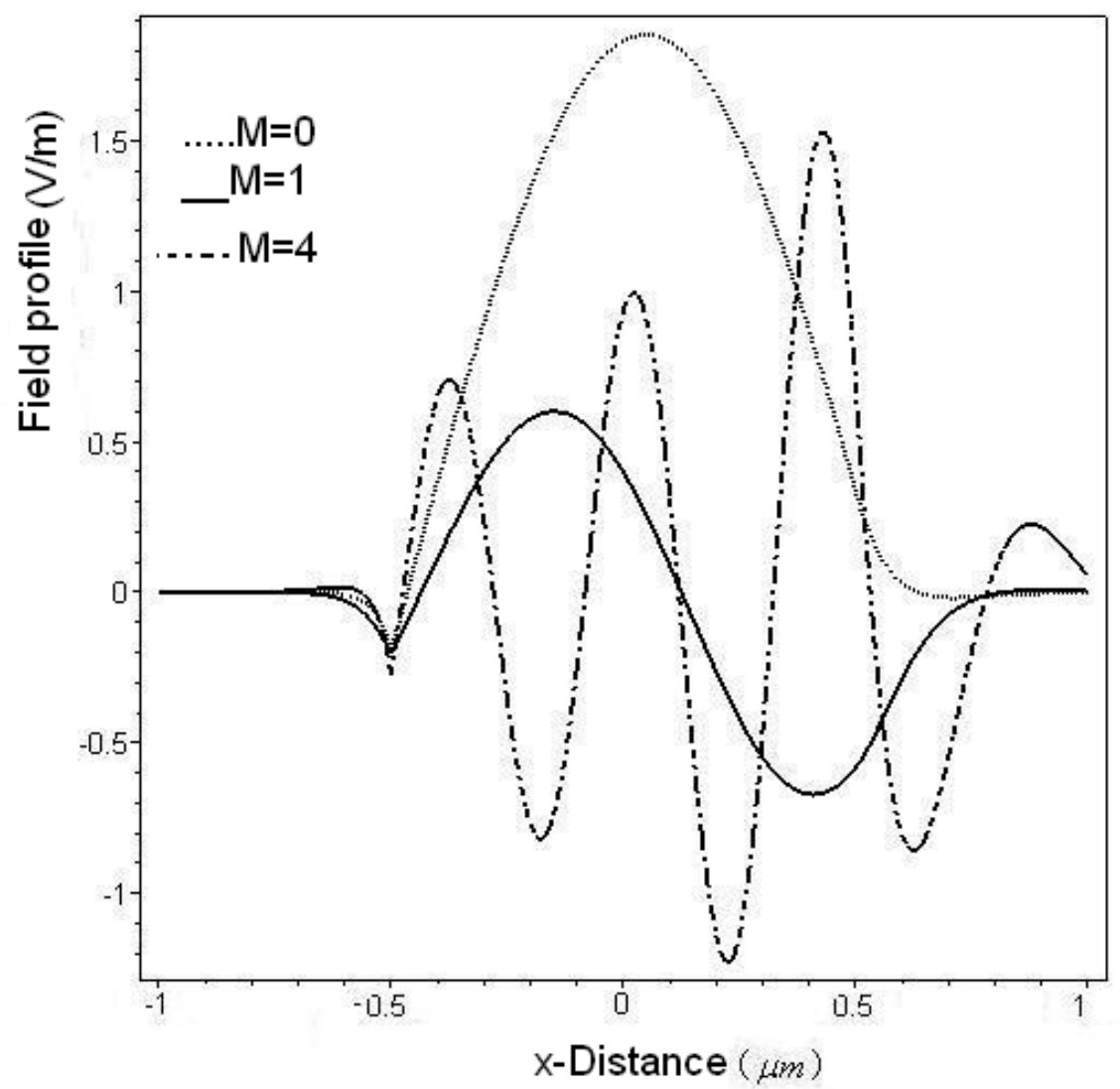

Fig. 2 Electric field profile (normalized to unit amplitude) of the $M=0$ (dotted), $M=1$ (solid) and $M=4$ (dot dashed) for LHM model, $\widetilde{\mathrm{n}}_{\mathrm{h}}=-3.74+\mathrm{i} 2, \widetilde{\mathrm{n}}_{\mathrm{s}}=1.26+\mathrm{i} 7.2, \mathrm{n}_{\mathrm{c}}=1, h=0.5 \mu \mathrm{m}, \lambda=1.9 \mu \mathrm{m}$.

Table 1 Propagation constants of TE mode $(0,1,4)$ solutions to LHM waveguide of $h=0.5 \mu m$. The parameters are of unit $\mu m^{-1}$.

\begin{tabular}{lllll}
\hline Mode & $k_{x}$ & $k_{z}$ & $\gamma_{c}$ & $\gamma_{s}$ \\
\hline 0 & $3.05-\mathrm{i} .0867$ & $12.06-\mathrm{i} 6.75$ & $6.97+\mathrm{i} 11.72$ & $6.86+\mathrm{i} 26.38$ \\
1 & $6.054-\mathrm{i} 0.17$ & $11.17-\mathrm{i} 7.23$ & $7.57+\mathrm{i} 10.8$ & $7+\mathrm{i} 25.8$ \\
4 & $15-\mathrm{i} 1$ & $5.46-\mathrm{i} 12$ & $13.4+\mathrm{i} 6$ & $8.2+\mathrm{i} 22$ \\
\hline
\end{tabular}


of increasing values of the longitudinal attenuation $\alpha_{\mathrm{z}}$ (imaginary part of $k_{z}$ ). $\alpha_{z}$ is important to the field of light trapping in thin films, as it represents the absorption length of a guided mode in the structure. Negative $\alpha_{z}$ means loss of wave power from the structure while positive $\alpha_{z}$ means absorption of wave power by the structure. By increasing $\alpha_{z}$ to the values of $(-6.75,-7.23,-12)$, the modes have negative absorption lengths and loss of the power from the film. For $\mathrm{M}=4$ absorption of the wave is achieved in LHM film as well as a dramatic evanescent decay in the substrate region $(-1 \prec x \prec-0.5)$ as shown by $\gamma_{s}$ values. In cladding region $(0.5 \prec x \prec 1)$, the electric field increases by mode's order. This is because of increasing the real part of the propagation number $\gamma_{c}$ to the values of $(6.97,7.57,13.4)$ for $\mathrm{M}$ $=0,1,4$ respectively. In Fig. 3 we plot electric field profile (normalized to unit amplitude) for the modes of lossy LHM waveguide (i.e. $\mathrm{M}=0, \mathrm{M}=1$ and $\mathrm{M}=$ 4) for the film thickness $h=0.3 \mu \mathrm{m}$. It is observed that as $h$ decreases, both modes 0,1 keep the same behavior as in Fig. 2 but mode 4 attains high normalized field of value (4) where it has positive attenuation $\alpha_{z}$ of value (23.7) as shown by Table 2 . In cladding region, the electric field of mode 4 increases sharply to the value of (30) which leads to high absorption in the cladding where most of the power is wasted in the cladding. Fig. 4 describes the variation of mode attenuation along z-axis $\alpha_{z}$ with mode's order for different LHM thickness. It is noticed that at $h=0.5 \mu \mathrm{m}$, high order modes generally have more loss than low-order modes since the mode attenuation along z-axis $\alpha_{z}$ increases to the values of $(-6.75,-7.23$ to -18.88$)$ with the mode's number of $(0,1$ to 6$)$. Besides that, the attenuation increases to negative values by thickness decrease to the values $(0.7,0.5,0.3) \mu m$ but at $h=0.3 \mu \mathrm{m}$, the modes of order $(4,5,6)$ attain high positive attenuation which means these modes have larger absorption lengths and they are better absorber than the others. It is worth to notice that LHM of $h=0.3 \mu \mathrm{m}$ is appropriate for solar cell applications. Figs. 5a and $5 b$, display a comparison of normalized electric field profile of $\mathrm{M}=3$ mode of LHM with RHM (amorphous silicon) and with a metal, at $\lambda=1.9 \mu \mathrm{m}$, and $h=0.3 \mu \mathrm{m}$. The refractive index of the LHM is $n_{h}=-3.74+i 2[20,21]$ and that is of amorphous silicon film (RHM) $[20,21]$ is $n_{f}=4.9+i 0.3$ and that is of metal film is assumed to be $n_{m}=-100+i 0.3$. The computed wave numbers for mode $\mathrm{M}=3$ are summarized in Table 3 . It is shown that the normalized electric field of LHM is amplified through the film to the value of $(3 \mathrm{~V} / \mathrm{m})$ and continues in the cladding. In RHM film, no amplifying of the field occurred while in the cladding it reaches much greater than $(-8 \mathrm{~V} / \mathrm{m})$ lead to the power leakage in the cladding. This is because $\alpha_{z}$ of RHM is $(+5.99)$ while for LHM it is (-15.87) and of metal (-0.99) as shown by Table 3. However, LHM structure guides the power better than RHM or metal one since LHM structure guides the power through the film more than wasting it in the cladding as in RHM structure. For arbitrary LHM, the frequency-dependent permittivity is described by the Drude medium model as Ref. [25].

$$
\varepsilon=\varepsilon_{\text {latice }}-\frac{\omega_{p}^{2}}{\omega^{2}+i \omega \gamma}
$$

where $\omega$ is the angular frequency, $\varepsilon_{\text {lattice }}$ is the lattice permittivity, $\omega_{p}=1.2 \times 10^{16} \mathrm{rad} / \mathrm{s}, \gamma=1.2 \times 10^{14} \mathrm{rad} / \mathrm{s}, \omega_{p}$ is the effective plasma frequency and $\gamma$ is the electric damping factor, $\varepsilon_{\text {lattice }}=9.1$. The calculations are performed for electromagnetic radiations in the visible regions at wavelength 600,700 to $900 \mathrm{~nm}$. In this frequency band the real parts of refractive indices of arbitrary LHM according to Eq. (21) are -2.338 , -3.274 to -4.86 while the permeability of LHM is assumed to be -1 . As shown by Fig. 6 longitudinal attenuations are computed and illustrated as a function of wavelength of the incident waves in the visible 

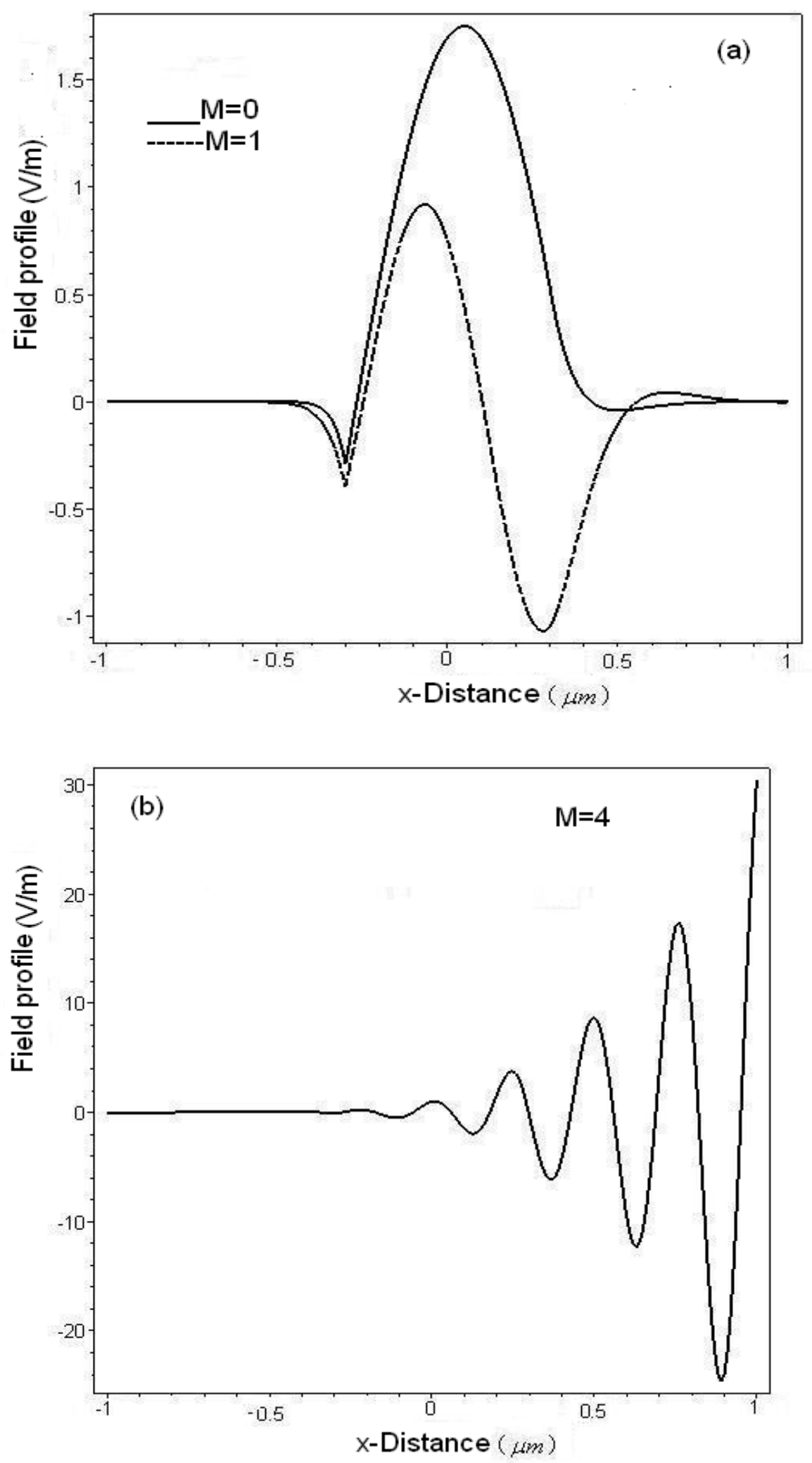

Fig. 3 (a) Electric field profile (normalized to unit amplitude) of the $M=0, M=1$ and (b) $M=4$ mode for LHM model, $\widetilde{\mathrm{n}}_{\mathrm{h}}=-3.74+\mathrm{i} 2, \widetilde{\mathrm{n}}_{\mathrm{s}}=1.26+\mathrm{i} 7.2, \mathrm{n}_{\mathrm{c}}=1, h=0.3 \mu \mathrm{m}, \lambda=1.9 \mu \mathrm{m}$. 
Table 2 Propagation constants of TE mode $(0,1,4)$ solutions to LHM waveguide of $h=0.3 \mu m$. The parameters are of unit $\mu m^{-1}$.

\begin{tabular}{lllll}
\hline Mode & $k_{x}$ & $k_{z}$ & $\gamma_{c}$ & $\gamma_{s}$ \\
\hline 0 & $4.94-\mathrm{i} 0.222$ & $11.558-\mathrm{i} 6.98$ & $7.32+\mathrm{i} 11.17$ & $6.94+\mathrm{i} 26$ \\
1 & $9.72-\mathrm{i} 0.55$ & $9.18-\mathrm{i} 8.32$ & $9.3+\mathrm{i} 8.78$ & $7.35+\mathrm{i} 24.6$ \\
4 & $26.4-\mathrm{i} 5.5$ & $2.667+\mathrm{i} 23.7$ & $24.45+\mathrm{i} 3.34$ & $13.95+\mathrm{i} 12.97$ \\
\hline
\end{tabular}

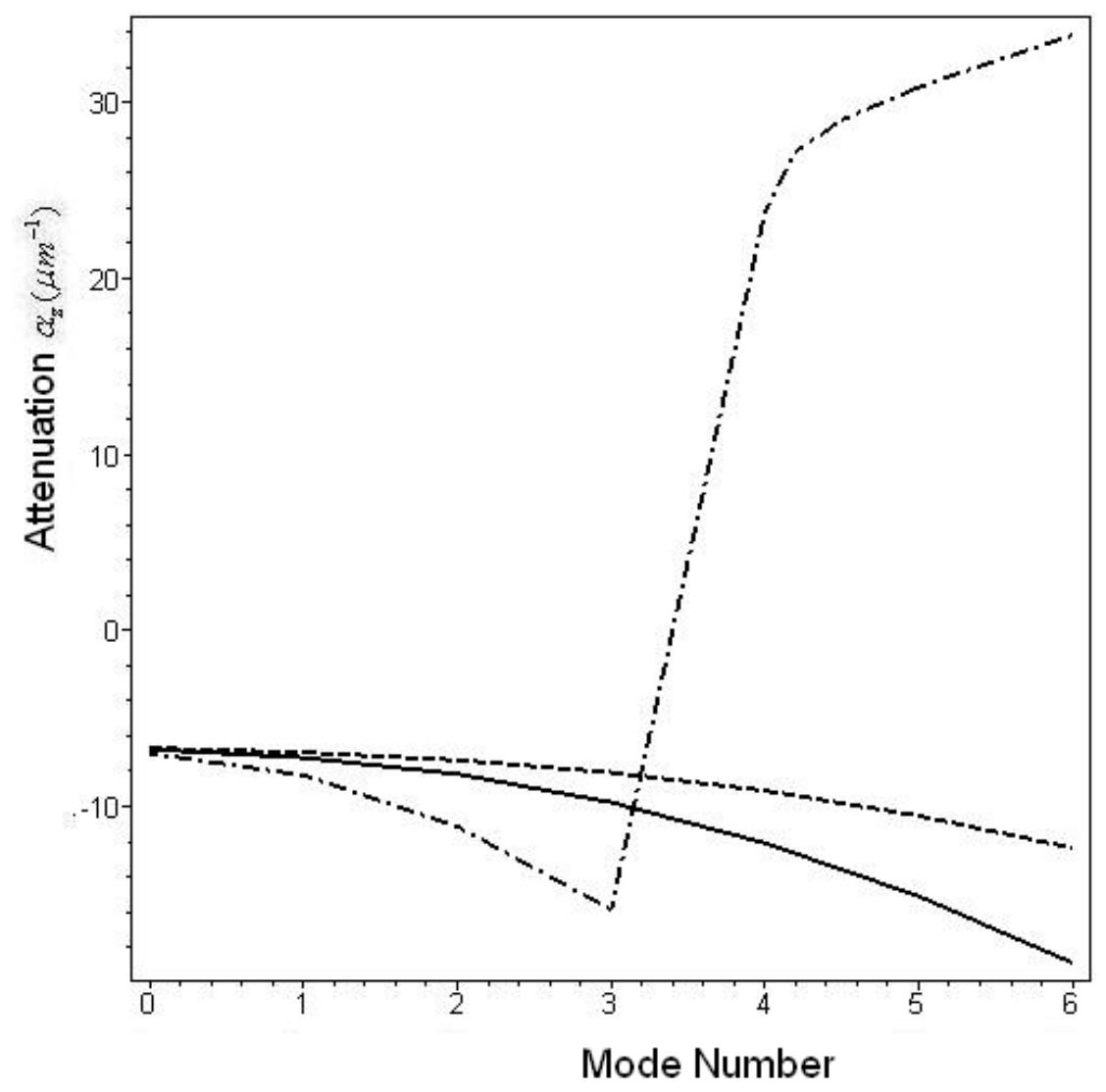

Fig. 4 Longitudinal attenuation coefficient $\left(\alpha_{z}\right)$ versus mode's number for different LHM thicknesses. $h=0.7 \mu m$ (dashed), $h=0.5 \mu \mathrm{m}$ (solid), $h=0.3 \mu \mathrm{m}$ (dot dash) LHM model, $\widetilde{\mathrm{n}}_{\mathrm{h}}=-3.74+\mathrm{i} 2, \widetilde{\mathrm{n}}_{\mathrm{s}}=1.26+\mathrm{i} 7.2, \quad \mathrm{n}_{\mathrm{c}}=1$, $\lambda=1.9 \mu \mathrm{m}$.

regions for TE mode's number $\mathrm{M}=(0,1,3)$ of arbitrary LHM model at $h=0.3 \mu \mathrm{m}$. It is observed that the attenuation in this frequency band increases to negative values with the wavelength decrease and mode's number increase. At wavelength $(600 \mathrm{~nm})$ $\alpha_{z}$ increases to the values of $(-21.2,-21.94,-23.25$, -25.2) $\mu \mathrm{m}^{-1}$ by mode's number $\mathrm{M}$ increase to $(0,1,2,3)$. This implies the best absorption is attained at longer wavelengths and for lower order modes. In Fig. 7, we explore the effect of the film thickness on the mode attenuation $\alpha_{\mathrm{z}}$ for arbitrary LHM model for $\mathrm{M}=0$. It has been noticed that the attenuation increases to negative values with thickness decrease. It means that if the film becomes thinner, the mode will suffer more loss through the wave propagation in the considered structure. 

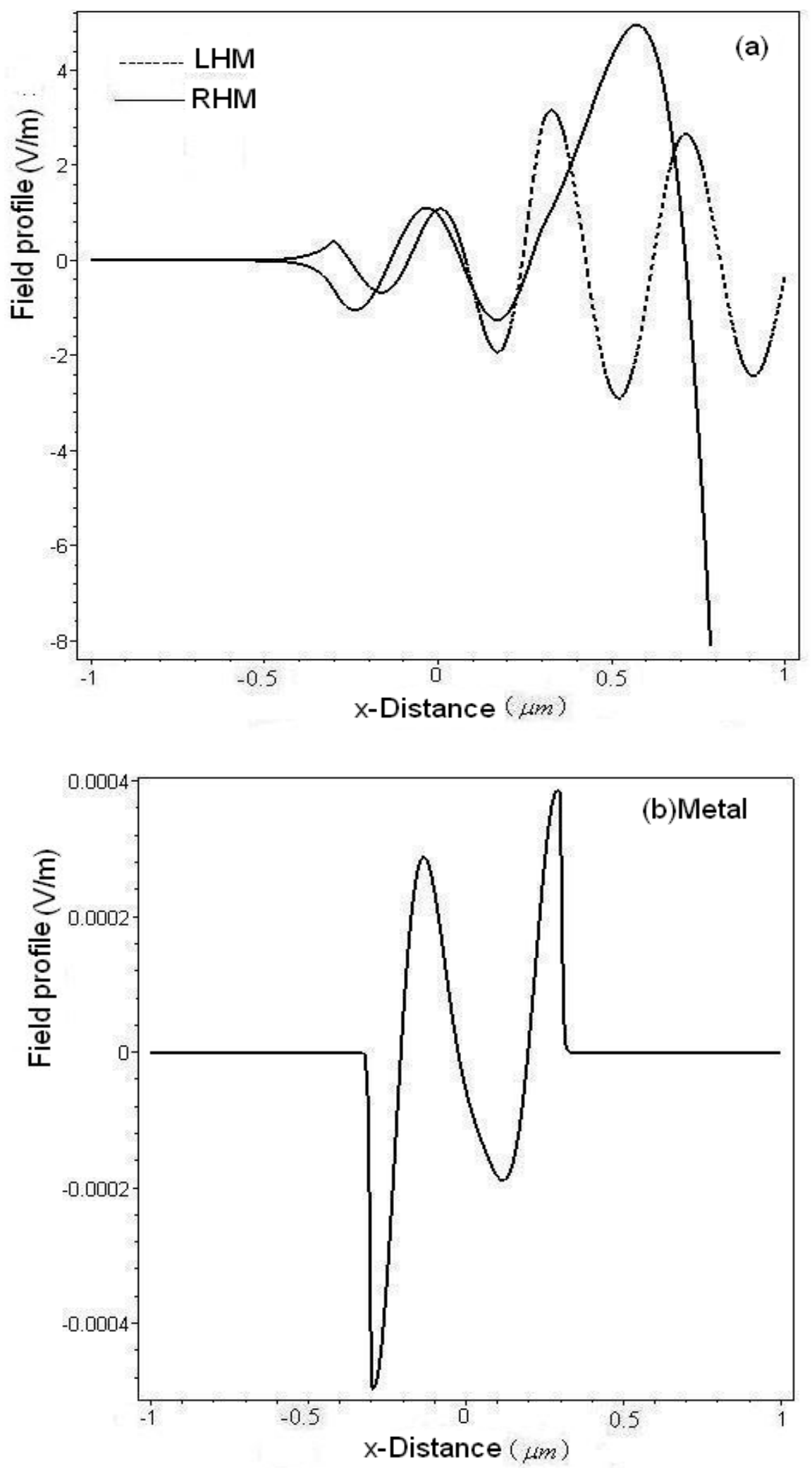

Fig. 5 (a) Electric field profile of the $\mathbf{M}=\mathbf{3}$ mode at $h=0.3 \mu \mathrm{m}, \lambda=1.9 \mu \mathrm{m}, \tilde{n}_{s}=1.26+\mathrm{i} .2$, $\mathrm{n}_{\mathrm{c}}=1$, where $\widetilde{n}_{h}=-3.74+\mathrm{i} 2$ for LHM, $n_{f}=4.9+i 0.3$ for RHM, and (b) for metal $n_{\mathrm{m}}=-100+i 0.3$. 
Table 3 Propagation constants of TE mode $(M=3)$ solutions to LHM, RHM and metal waveguide of $h=0.3 \mu m$. The parameters are of unit $\mu m^{-1}$.

\begin{tabular}{lllll}
\hline Film & $k_{x}$ & $k_{z}$ & $\gamma_{c}$ & $\gamma_{s}$ \\
\hline LHM & $19.38-\mathrm{i} 3.84$ & $0.45-\mathrm{i} 15.87$ & $18.69+\mathrm{i} 4.3$ & $9.97+\mathrm{i} 18.15$ \\
RHM & $16.4-\mathrm{i} 1.007$ & $5.43+\mathrm{i} 5.99$ & $6.62-\mathrm{i} 2.4$ & $3.59+\mathrm{i} 23.13$ \\
Metal & $20.73+0.7 \times 10^{-3}$ & $330-\mathrm{i} 0.99$ & $0.99+\mathrm{i} 330$ & $1.29+\mathrm{i} 330.8$ \\
\hline
\end{tabular}

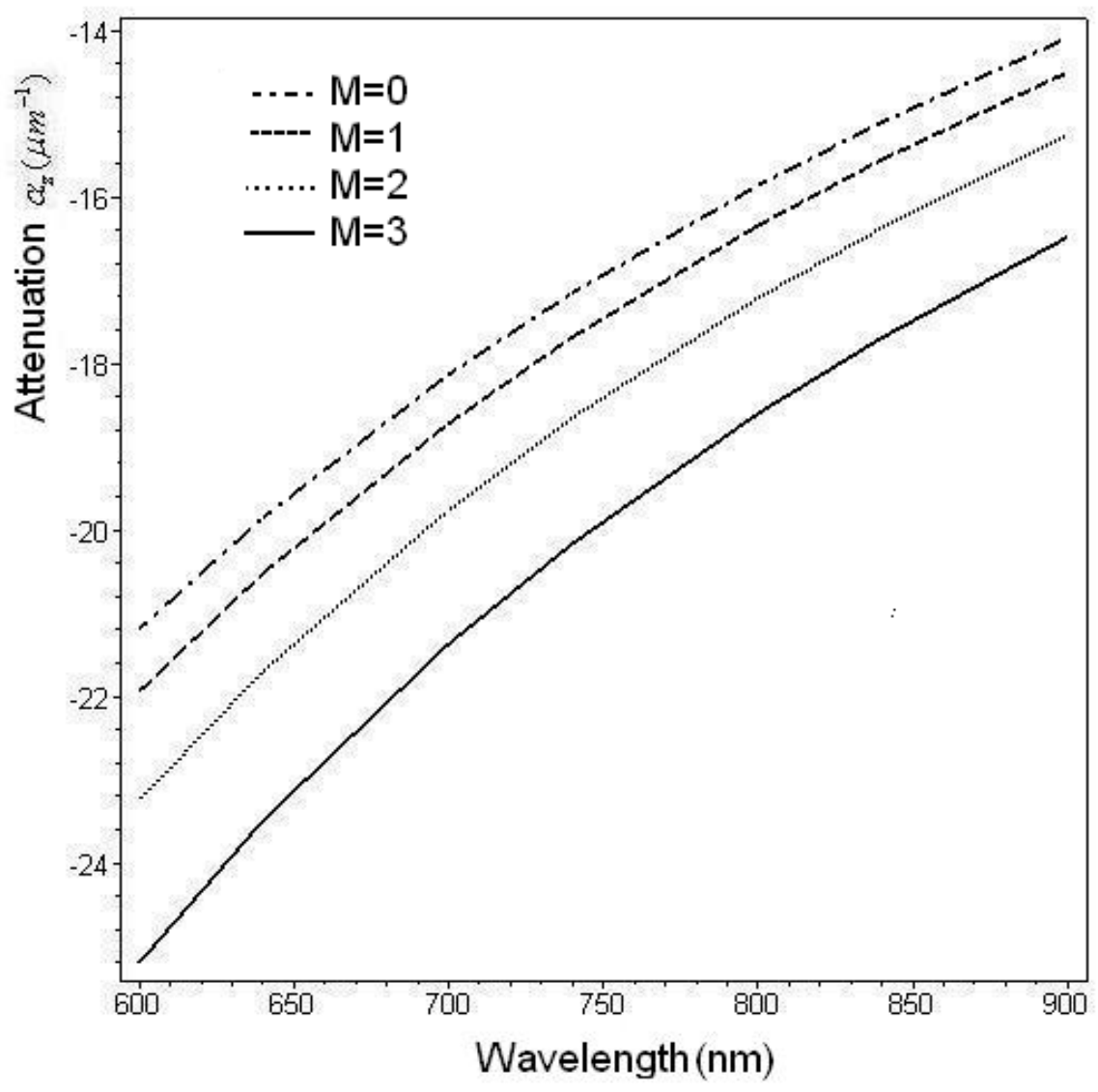

Fig. 6 Longitudinal attenuation coefficient $\left(\alpha_{z}\right)$ of TE waves versus wavelength for mode's number $\mathbf{M}=\mathbf{0}$ (dash dot), $\mathbf{M}=$ 1 (dashed), $\mathbf{M}=2$ (dotted), $\mathbf{M}=3$ (solid) of arbitrary LHM model for, $h=0.3 \mu \mathrm{m}, n_{c}=1, \widetilde{n}_{s}=1.26+\mathrm{i} 7.2$. 


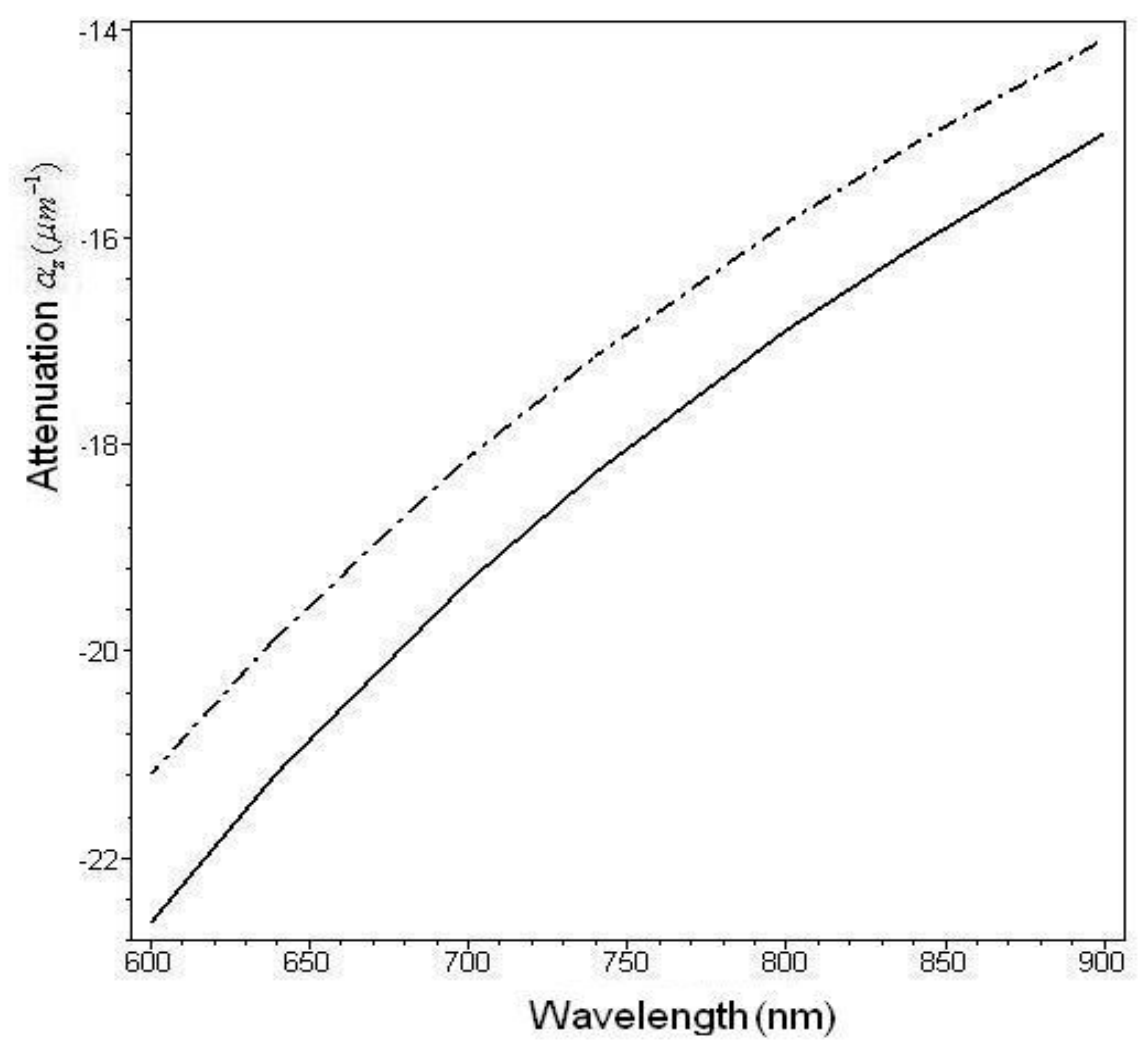

Fig. 7 Longitudinal attenuation coefficient $\left(\alpha_{z}\right)$ of TE waves versus wavelength of mode's number $\mathbf{M}=\mathbf{0}$ for $h=0.3 \mu \mathrm{m}$ (dash dot), $h=0.1 \mu \mathrm{m}$ (solid) of arbitrary LHM model $n_{c}=1, \tilde{n}_{s}=1.26+\mathrm{i} 7.2$.

\section{Conclusions}

We derived and simulated the modal dispersion relation and attenuation of TE modes in an asymmetric slab waveguide constructed from lossy thin LHM film sandwiched between a lossy substrate and coverd by a losseless dielectric cladding. The numerical solutions showed that guided high order modes in thinner films, generally have more loss of power than low-order modes since the mode attenuation along $z$-axis $\alpha_{z}$ increases to negative values as the mode's number increases and the film thickness decrease. For LHM, at incident wavelength $=1.9 \mu \mathrm{m}$ and at thickness $h=0.3 \mu \mathrm{m}$, the modes of order $(4,5,6)$ attain high positive attenuation which means these modes have larger absorption lengths and they are better absorber than the others which implies this LHM is appropriate for solar cell applications. For arbitrary LHM, the best absorption is attained at longer wavelengths in the considered frequency band and for lower order modes at wider films. The obtained results may be used to refine the understanding of any related applications that may be modeled using the light trapping in thin film solar cells.

\section{Acknowledgements}

The authors acknowledge financial support from BMBF through the NANOWAFSOL project, Germany.

\section{References}

[1] Cai, W., and Shalaev, V. 2010. Optical Metamaterials. New York: Springer.

[2] Govind, D., and Ramakrishna, S. A. 2012. "Design of Highly Absorbing Metamaterials for Infrared Frequencies." Optics Express (16): 17503.

[3] Pendry, J. B., Schurig, D., and Smith, D. R. 2006. "Controlling Electromagnetic Fields." Science 312: 1777-9. 
[4] Ghosh, S., Bhattacharyya, S., Kaiprath, Y., and Srivastava, K. 2014. "Bandwidth-Enhanced Polarization-Insensitive Microwave Metamaterial Absorber and Its Equivalent Circuit Model.” Journal of Applied Physics 115 (10): 4184.

[5] Wang, B. X., Zhai, X., Wang, G. Z., Huang, W. Q., and Wang, L. L. 2015. "Design of a Four-Band and Polarization-Insensitive Terahertz Metamaterial Absorber.” IEEE Photonics Journal 7 (1): 4600108.

[6] Liu, Z., Yi Huang, C., Liu, H., Zhang, X., and Lee, C. 2013. "Resonance Enhancement of Terahertz Metamaterials by Liquid Crystals/Indium Tin Oxide Interfaces." Optics Express 21 (5): 6519-25.

[7] Liu, X., and Padilla, W. J. 2013. "Dynamic Manipulation of Infrared Radiation with MEMS Metamaterials." Advanced Opt. Materials 1 (8): 559-62.

[8] Pitchappa, P., Pei Ho, C., Kropelnicki, P., Singh, P. N., Kwong, D., and Lee1, C. 2014. "Switchable Near Infrared Complementary Metamaterial Absorber." App. Phys. Letters 104: 201114.

[9] Ubeid, M. F., Shabat, M. M., and Schaadt, D. M. 2016. "Wide-Angle and Wavelength Independent Perfect Absorption at Metamaterial Surfaces." Romanian Reports in Physics 68 (2): 725-35.

[10] Hao, J., Wang, J., Liu, X., Padilla, W. J., Zhou, L., and Qiu, M. 2010. "High Performance Optical Absorber Based on a Plasmonic Metamaterial.” Appl. Phys. Lett. 96: 251104.

[11] Shrekenhamer, D., Watts, C. M., and Padilla, W. J. 2013. "Terahertz Single Pixel Imaging with an Optically Controlled Dynamic Spatial Light Modulator." Optics Express 21 (10): 12507-18.

[12] Meng, L., Zhao, D., Ruan, D., Z., Li, Q., Yang Y., and Qiu, M. 2014. "Optimized Grating as an Ultra-narrow Band Absorber or Plasmonic Sensor." Optics Letters 39 (5): 1137-40.

[13] Cong, L., Tan, S., Yahiaoui, R., Yan, F., Zhang, W., and Singh, R. 2015. "Experimental Demonstration of Ultrasensitive Sensing with Terahertz Metamaterial
Absorbers." Applied Phys. Letters 106: 031107.

[14] Maier, T., and Brueckl, H. 2010. "Multispectral Microbolometers for the Mid Infra-Red." Opt. Lett. 35: 3766-8.

[15] Shabat, M. M., and Ubeid, M. 2014. "Antireflection Coating at Metamaterial Waveguide Structures for Solar Energy Applications." Energy Procedia 50: 314-21.

[16] Hamouche, H., Shabat, M. M., and Schaadt, D. M. 2016. "Multilayer Solar Cell Waveguide Structures Containing Metamaterials." Superlattices and Microstructures 101: 633-40.

[17] Hamouche H., and Shabat, M. M. 2016. "Enhanced Absorption in Silicon-Metamaterials Waveguide Structure." Applied Physics A 122 (7): 1-7.

[18] Wu, C., Neuner, B., John, J., Milder, A., Zollars, B., Savoy, S., and Shvets, G. 2012. "Metamaterial-Based Integrated Plasmonic Absorber/Emitter for Solar Thermo-Photovoltaic Systems.” J. Opt. 14: 024005.

[19] Landy, N. I., Sajuyigbe, S., Mock, J. J., Smith, D. R., and Padilla, W. J. 2008. "Perfect Metamaterial Absorber." Phys. Rev. Lett. 100: 207402.

[20] Nagel, J. R., Blair, S., and Scarpulla, M. A. 2011. "Exact Field Solution to Guided Wave Propagation in Lossy Thin Films.” Optics Express 19 (21): 20159.

[21] Nagel, J. R., Blair, S., and Scarpulla, M. A. 2012. "Exact Field Solution to Guided Wave Propagation in Lossy Thin Films." Proc. of SPIE 8256 (2012): 825606-1.

[22] Zhang, S., Fan, W., Malloy, K. J., and Brueck, S. R. J. 2005. "Near-Infrared Double Negative Metamaterials." Optics Express 13 (13): 4922-30.

[23] Mousa, H. M., and Shabat, M. M. "Electromagnetic Guided Waves in a Metamaterial Magnetic Waveguide Structure.” Int. J. Modern Physics B 25 (32).

[24] Mousa, H. M., and Shabat, M. M. 2013. "TM Waves in Cylindrical Superlattices (LANS) Bounded by Left Handed Materials." App. Phys. A 111: 1057-63.

[25] TH, Isaac. 2009. "Tunable Plasmonic Structures for Terahertz Frequencies." Ph.D. thesis, University of Exeter, UK. 